\title{
Techniques in Endourology
}

\section{Pediatric Robot-Assisted Laparoscopic Excision of Urachal Cyst and Bladder Cuff*}

\author{
Jonathan Yamzon, M.D., ${ }^{1}$ Paul Kokorowski, M.D., ${ }^{1}$ Roger E. De Filippo, M.D., ${ }^{1,2,3}$ Andy Y. Chang, M.D., ${ }^{1,2,3}$ \\ Brian E. Hardy, M.D., ${ }^{1,3}$ and Chester J. Koh, M.D. ${ }^{1,2,3}$
}

\begin{abstract}
Urachal cysts are the most common urachal anomaly in the pediatric population. There is an increasing body of literature documenting successful management of urachal cysts using laparoscopic techniques. There may be an advantage, however, with the use of robot-assisted laparoscopy for reconstructive cases. We describe the techniques used for robot-assisted laparoscopic excision of a urachal cyst and bladder cuff with bladder repair in a female child. This approach is a safe and effective option for the minimally invasive management of pediatric urachal cysts.
\end{abstract}

\section{Introduction}

$\mathbf{T}$ HE URACHUS, a three-layered canal that connects the allantois to the fetal bladder, usually obliterates in the fifth month of gestation. Urachal anomalies in the pediatric population are rarely associated with malignancy. Urachal cysts represent up to $54 \%$ of pediatric urachal anomalies. ${ }^{1-4}$

Several recent reports have described successful management of urachal anomalies using minimally invasive techniques. ${ }^{5-11}$ For pediatric patients, minimally invasive approaches for urachal anomalies have been limited to traditional laparoscopic techniques. ${ }^{7-9}$ Robot assistance in laparoscopic surgery is increasingly being used for reconstructive procedures that are needed after extirpative surgery. To date, robot-assisted laparoscopy for urachal anomalies has only been described in adult patients. ${ }^{5,6}$ In this report, we present the techniques used in pediatric robot-assisted laparoscopic excision of a urachal cyst with a bladder cuff, with subsequent bladder repair.

\section{Technique}

We performed a robot-assisted laparoscopic excision of a previously infected urachal cyst in a 4-year-old girl who ini- tially presented to the emergency department with midline abdominal pain. She was otherwise noted to be healthy, and there was no history of umbilical drainage. A CT scan of the abdomen and pelvis revealed an anterior midline urachal cyst located just superior to the bladder that measured 2.7 $\mathrm{cm}$ in its greatest diameter and which was associated with local inflammatory changes (Fig. 1). Her abdominal pain resolved after 2 weeks of oral antibiotics. After 2 months of observation to allow the inflammatory changes to subside, options for management of the urachal cyst were discussed with the family. The family elected to proceed with robotassisted laparoscopic excision of the urachal cyst and bladder cuff with subsequent bladder repair.

\section{Patient positioning and robot setup}

Cystoscopy and cystography are performed initially to identify a possible connection from the bladder to the urachal cyst. The patient is placed in the supine position, padded at all pressure points, and secured to the table using wide tape. The Trendelenberg position is used to allow the abdominal contents to fall in a cephalad manner away from the pelvis. A Foley catheter is placed in a sterile fashion to allow for saline installation to achieve intermittent bladder distension.

\footnotetext{
${ }^{1}$ Department of Urology, University of Southern California Keck School of Medicine, Los Angeles, California.

${ }^{2}$ Department of Surgery, Huntington Memorial Hospital, Pasadena, California.

${ }^{3}$ Division of Pediatric Urology, Childrens Hospital Los Angeles, Los Angeles, California.

*A video demonstrating this technique is available on the enclosed CD-ROM and online at www.liebertpub.com/end.
} 


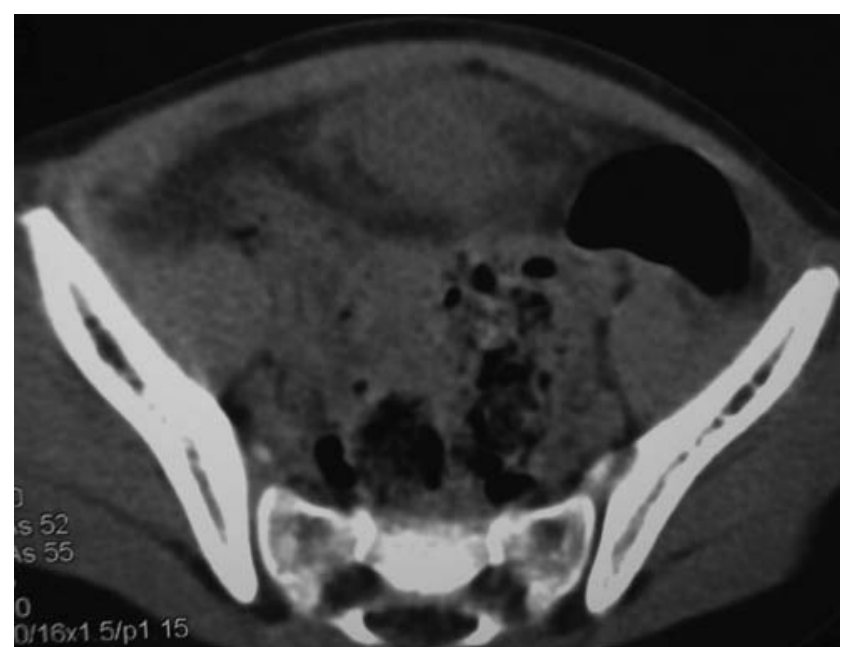

FIG. 1. A CT scan of the abdomen shows a midline anterior cystic mass just superior to the bladder with local inflammation consistent with an inflamed urachal cyst.

Peritoneal access is obtained using the open Hasson technique, ${ }^{12}$ with placement of the $12-\mathrm{mm}$ camera port in the midline using a vertical incision halfway between the umbilicus and the xyphoid process. Subsequent pneumoperitoneum of 12 to $15 \mathrm{~mm} \mathrm{Hg}$ is applied, and the laparoscope is inserted. Two additional ports ( $5 \mathrm{~mm}$ or $8 \mathrm{~mm}$ ) are inserted under direct vision at the anterior axillary lines just above the level of the umbilicus (Fig. 2). A box stitch is applied to all ports to secure them in place and for use during the port closures. ${ }^{13}$

The Da Vinci surgical robot system (Intuitive Surgical, Sunnyvale, CA) is brought over the legs from the caudal direction, and the robotic arms are engaged to the ports. Hook cautery or the hot scissors are used for dissection, and DeBakey forceps are used for grasping. The 30-degree angled camera lens is positioned upward to visualize the bladder and anterior abdominal wall.

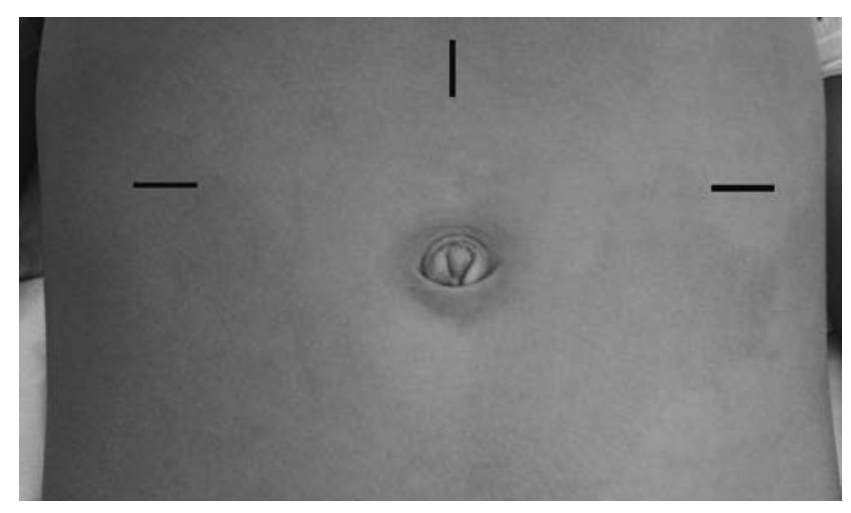

FIG. 2. A 12-mm camera port is placed through a vertical incision at midline halfway between the umbilicus and the xyphoid process; 5- or 8-mm ports are placed through horizontal incisions at the anterior axillary lines just superior to level of umbilicus

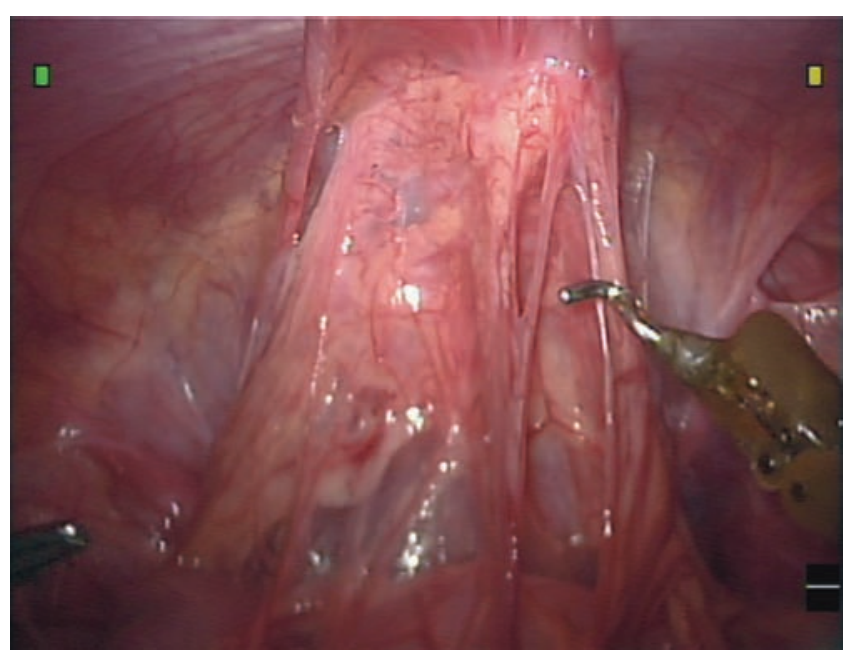

FIG. 3. Omental adhesions cover the urachal cyst.

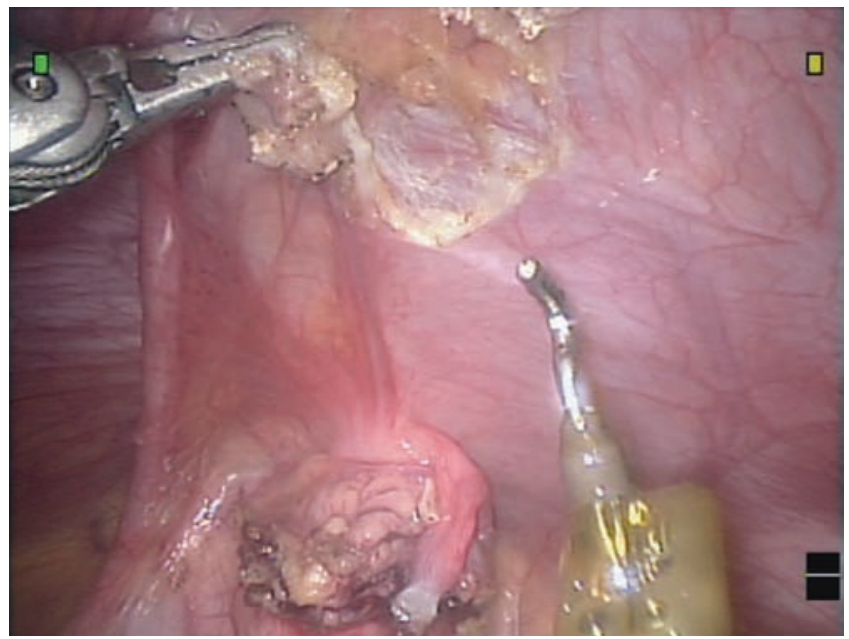

FIG. 4. Dissection of the urachus from the anterior abdominal wall occurs after division of the right obliterated umbilical artery.

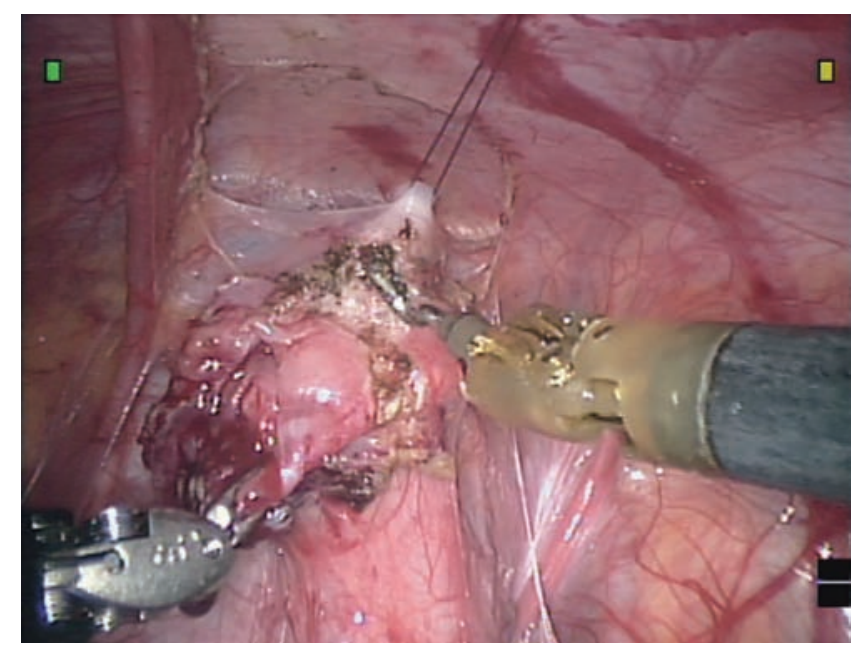

FIG. 5. With the hitch stitch providing countertraction, the urachal cyst and bladder cuff are excised from the rest of the bladder. 


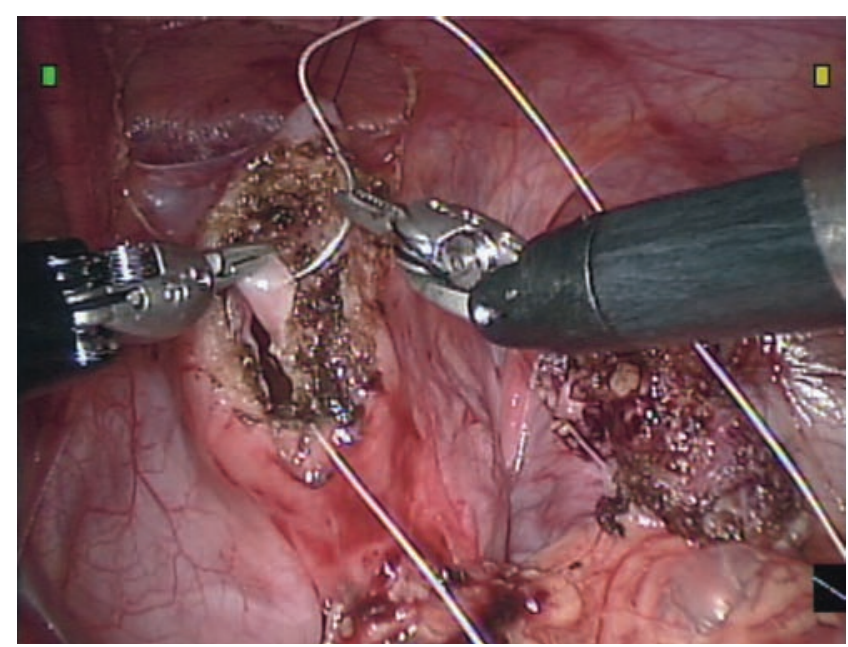

FIG. 6. Two layer closure of the bladder opening is used after excision of the urachal cyst and bladder cuff.

\section{Initial approach}

Dissection begins with lysis of the omental adhesions, which are often adherent to the cyst (Fig. 3). Once the adhesions are freed, one or both of the obliterated umbilical arteries are cauterized and divided to allow access to the anterior bladder wall (Fig. 4). The urachal remnant is cauterized and divided at its superior end near the umbilicus and then carefully dissected from the anterior abdominal wall using cautery. The dissection is carried down to the dome of the bladder, and the anterior bladder wall is mobilized from the anterior abdominal wall as well. The bladder can be distended partially or fully to assist with this dissection.

\section{Hitch stitch}

After mobilization of the urachal remnant, a holding stitch is passed through the abdominal wall above the bladder, then passed through the anterior bladder wall, and then out through the abdominal wall next to its initial insertion site, where it is clamped at the level of the skin (Fig. 5). The tension on this hitch stitch can then be adjusted to allow for better visualization of the surgical area, while providing countertraction during the cyst excision, as well as stability during suturing. Alternatively, an internal holding suture can be used to temporarily attach the anterior bladder wall to the anterior abdominal wall without passing the suture through the entire wall thickness.

\section{Urachal cyst and bladder cuff excision}

The urachal cyst and the bladder cuff are excised circumferentially using electrocautery; the hitch stitch and bladder distension via the Foley catheter can be adjusted as needed. A bladder opening is created once the urachal cyst and bladder cuff are excised as a single unit. Spillage of the bladder irrigant and urine is minimized because of the hitch stitch. The bladder defect is closed in a running fashion with two separate layers of 3-0 absorbable suture (Fig. 6). After closure, the bladder is distended via the Foley catheter to ensure a watertight anastomosis.
Any spilled urine or blood is aspirated from the field. Once the robot is disengaged, the specimen is placed within a laparoscopic pouch and removed under direct visualization through one of the arm port sites after the arm port is removed. The other ports are removed under direct visualization, and the abdominal wall defects are closed in layers.

Foley catheter drainage for 24 to 48 hours is sufficient for complete bladder closure, similar to ureteral reimplantation surgery. Pathologic examination confirmed the presence of a benign urachal cyst with extensive acute and chronic inflammation.

\section{Role in Urologic Practice}

Minimally invasive robot-assisted surgery is a safe and viable option in a wide range of surgical conditions in children. ${ }^{14-16}$ Robot-assisted surgery is increasingly being applied to pediatric cases that involve urologic reconstruction and are of greater complexity. ${ }^{17}$ For pediatric urachal anomalies, minimally invasive management of pediatric urachal anomalies has so far been limited to traditional laparoscopic techniques. We present the first description of the techniques for a pediatric robot-assisted laparoscopic excision of a urachal cyst and bladder cuff with bladder repair.

Previously published reports of successful outcomes of laparoscopic surgery for urachal anomalies have alluded to the potential advantages in children, including shorter hospital stays, decreased pain medication usage, and improved cosmesis, all of which should apply to robot-assisted laparoscopic surgery as well. ${ }^{18}$ Robot-assisted laparoscopic surgery offers pediatric surgical specialists and their patients an effective minimally invasive tool for increased precision during complex pediatric surgical procedures that require reconstruction, such as the excision of a urachal cyst and bladder cuff with bladder repair.

\section{Disclosure Statement}

No competing financial interests exist.

\section{References}

1. Ashley RA, Inman BA, Routh JC, Rohlinger RA, Husmann DA, Kramer SA. Urachal anomalies: A longitudinal study of urachal remnants in children and adults. J Urol 2007;178: 1615-1618.

2. McCollum MO, Macneily AE, Blair GK. Surgical implications of urachal remnants: Presentation and management. J Pediatr Surg 2003;38:798-803.

3. Bauer SB, Retik AB. Urachal anomalies and related umbilical disorders. Urol Clin North Am 1978;5:195-211.

4. Cilento BG Jr, Bauer SB, Retik AB, Peters CA, Atala A. Urachal anomalies: Defining the best diagnostic modality. Urology 1998;52:120-122.

5. Madeb R, Knopf JK, Nicholson C, et al. The use of robotically assisted surgery for treating urachal anomalies. BJU Int 2006;98:838-842.

6. Yano H, Iwazawa T, Monden T. Excision of urachal sinus with use of a voice-controlled laparoscope. J Laparoendosc Adv Surg Tech A 2003;13:45-49.

7. Khurana S, Borzi PA. Laparoscopic management of complicated urachal disease in children. J Urol 2002;168:1526-1528.

8. Yohannes P, Bruno T, Pathan M, Baltaro R. Laparoscopic radical excision of urachal sinus. J Endourol 2003;17:475-479. 
9. Kojima Y, Hayashi Y, Yasui T, Itoh Y, Maruyama T, Kohri $\mathrm{K}$. Laparoscopic management for urachal cyst in 9-year-old boy. Int Urol Nephrol 2007;39:771-774.

10. Cadeddu JA, Boyle KE, Fabrizio MD, Schulam PG, Kavoussi LR. Laparoscopic management of urachal cysts in adulthood. J Urol 2000;164:1526-1528.

11. Stone NN, Garden RJ, Weber H. Laparoscopic excision of a urachal cyst. Urology 1995;45:161-164.

12. Hasson HM. Open laparoscopy. Biomed Bull 1984;5:1-6.

13. Poppas DP, Bleustein CB, Peters CA. Box stitch modification of Hasson technique for pediatric laparoscopy. J Endourol 1999;13:447-450.

14. Najmaldin A, Antao B. Early experience of tele-robotic surgery in children. Int J Med Robot 2007;3:199-202.

15. Meehan JJ, Sandler A. Pediatric robotic surgery: A single-institutional review of the first 100 consecutive cases. Surg Endosc 2008;22:177-182.

16. Volfson IA, Munver R, Esposito M, Dakwar G, Hanna M, Stock JA. Robot-assisted urologic surgery: Safety and feasibility in the pediatric population. J Endourol 2007;21:1315-1318.

17. Passeroti CC, Nguyen HT, Eisner BH, Lee RS, Peters CA. Laparoscopic reoperative pediatric pyeloplasty with robotic assistance. J Endourol 2007;21:1137-1139.

18. Peters CA. Laparoscopic and robotic approach to genitourinary anomalies in children. Urol Clin North Am 2004;31:595-605.

Address reprint requests to: Chester J. Koh, M.D.

Division of Pediatric Urology

Childrens Hospital Los Angeles

4650 Sunset Blvd, Mailstop 114

Los Angeles, CA 90027

E-mail: ckoh@chla.usc.edu

Abbreviations Used
$\mathrm{CT}=$ computed tomography

DOI: $10.1089 /$ end.2008.0338a

\section{Editorial comment}

The authors should be congratulated for reporting the first application of the da $\operatorname{Vinci}^{\circledR}{ }^{\circledR}$ surgical system in a pediatric patient with urachal anomaly. There no doubt that a skilled laparoscopist can accomplish the same task without the use of such an intricate and very expensive computer system. However, this seemingly "entry-level" robotic case should prepare the minimally invasive surgeon for technically difficult and more complex cases such as robotic enterocystoplasty. The lessons learned from minor details of the operation during these "entry-level" cases, including port placements, use of the fourth arm for traction, or choices of instrumentation (e.g., Hook electrocautery vs. hot shears), all will instill confidence and build surgical experience, allowing the surgeon to tackle more difficult future cases with relative ease.

Having proctored more that three dozen robotic prostatectomies, radical cystectomies, and pyeloplasty in both academic and community hospital settings across the country, I have witnessed numerous avoidable pitfalls during these complex cases due to either lack of experience or sometimes enthusiasm in basic and "simple" laparoscopic and robotic cases that serve as the core foundation for more complicated ablative and reconstructive surgical procedures. Often, the ifirst da Vinci ${ }^{\circledR}$ prostatectomy" is the "first laparoscopic and robotic clinical case" to which the surgeon is exposed. This can create a level of frustration for both the surgeon and the staff that can result in very long operative time and more importantly, less than optimal clinical outcome.

Today, most reports in the literature addressing the da Vinci $^{\circledR}$ surgical system have evolved around prostatectomies, radical cystectomies, and most recently, partial nephrectomies. However, in daily clinical practice, as often times as the opportunity arises, urologists with access to the da Vinci ${ }^{\circledR}$ surgical system should strive to identify the different suitable cases that will help to refine their "robotic" skills, while maintaining the advantages of minimally invasive surgery for the patient. This manuscript represents such a case!

Address reprint requests to: Paulos Yohannes, M.D. Creighton University Omaha, Nebraska

E-mail: Pyohannes@hotmail.com 